\title{
A Gestão do Conhecimento holística e a aderência do modelo Fivaz e Pretorius
}

\author{
The holistic Knowledge Management and adherence \\ of Fivaz and Pretorius model
}

Fábio CORRÊA ${ }^{1}$ (iD) 0000-0002-2346-0187

\section{Resumo}

A atual Gestão do Conhecimento é fundamentada no paradigma reducionista. No entanto, é premente a necessidade de avançar, seguindo os preceitos do paradigma holístico, sendo esta uma lacuna científica ainda não preenchida. Nesse sentido, esta pesquisa ajusta o gerenciamento do conhecimento à luz do paradigma holístico, de modo a elucidar o almejado pela Gestão do Conhecimento mediante esse padrão científico. Com essa fundamentação, têm-se por objetivo analisar a aderência do modelo de Fivaz e Pretorius à Gestão do Conhecimento holística, visando identificar se um modelo com essa envergadura foi atingido. A Análise de Conteúdo, de abordagem qualitativa, e a quantificação percentílica são os procedimentos metodológicos adotados para prover o exame do referido modelo. Por resultado, o modelo analisado adere-se parcialmente aos preceitos da Gestão do Conhecimento holística, permitindo inferir que a lacuna assinalada ainda permanece. A análise de outros modelos, seguindo os delineamentos metodológicos desenvolvidos nesta pesquisa, é uma oportunidade de futuras investigações.

Palavras-chave: Análise de conteúdo. Gestão do conhecimento. Gestão do conhecimento holística. Paradigma holístico.

\begin{abstract}
Knowledge Management is currently based on the reductionist paradigm. However, it is urgent to move forward and follow the holistic paradigm's precepts, a scientific gap that has not been fulfilled yet. In this sense, this research adjusts knowledge management in light of the holistic paradigm, in order to elucidate the goal of the former through this scientific standard. Based on this rationale, this research aims to analyze the adherence of the Fivaz and Pretorius model to the holistic knowledge management, in order to identify if a model with this scale was reached. The methodological procedures adopted for the examination of such model are Content Analysis with a qualitative approach and percentage quantification. We concluded that the analyzed model adheres partially to the precepts of the holistic knowledge management, allowing to infer that the indicated gap still remains. The analysis of other models following the methodological delineations developed in this research, is an opportunity for future investigations.
\end{abstract}

Keywords: Content analysis. Knowledge management. Holistic knowledge management. Holistic paradigm.

\footnotetext{
1 Universidade FUMEC, Departamento de Pós-Graduação, Programa de Pós-Graduação em Sistemas de Informação e Gestão do Conhecimento. Av. Afonso Pena, 3880, Cruzeiro, 30130-009, Belo Horizonte, MG, Brasil. E-mail: <fabiocontact@gmail.com>.

Apoio: Fundação Mineira de Amparo à Pesquisa do Estado de Minas Gerais e Universidade FUMEC.

Artigo elaborado a partir da tese de F. CORRÊA, intitulada "A gestão do conhecimento holística: conformação de seus fatores, análise do presente e direcionamento para estudos futuros". Universidade FUMEC, 2018.

Recebido em 7 de janeiro de 2019 e aprovado em 27 de junho de 2019.
}

Como citar este artigo/How to cite this article

CORRÊA, F. A Gestão do Conhecimento holística e a aderência do modelo Fivaz e Pretorius. Transinformação, v. 32, e180089, 2020. http://dx.doi. org/10.1590/1678-9865202032e180089 


\section{Introdução}

Um paradigma é compreendido como um padrão de instrumentos metodológicos utilizados pela ciência para abordar seus problemas (Kuhn, 2018). O alicerce da ciência contemporânea foi e permanece sedimentado no paradigma reducionista, ou seja, no método cartesiano de reduzir um problema em menores partes para entendê-las de modo isolado do todo, visando, assim, alcançar a compreensão do fenômeno em sua completude (Crema, 2015; Pereira, 2017).

Capra (2014) demonstra, de forma eloquente, como esse paradigma está imbricado nas ciências. Em consonância com esses pesquisadores, Crema (2015) e Pereira (2017), demarcam que esse paradigma se tornou insuficiente para tratar os problemas da ciência moderna, pois, ao analisar as partes de forma isolada, as conexões entre essas secções são perdidas, acarretando a compreensão completa, mas não íntegra do fenômeno.

No âmbito da Gestão do Conhecimento (GC), os estudos de Holsapple e Joshi (1999), Rubenstein-Montano et al. (2001), Heisig (2009) e Fteimi (2015) assinalam que o paradigma holístico é uma alternativa para o desenvolvimento de modelos de gerenciamento do conhecimento, o que ajusta e determina a GC holística. Os pesquisadores supracitados não apontaram, no entanto, nenhum modelo holístico, revelando uma lacuna científica.

Mediante o exposto, esta pesquisa busca analisar a aderência do modelo de Fivaz e Pretorius (2015), à GC holística. A escolha desse modelo se dá pelo critério de temporalidade. A proposta de Fivaz e Pretorius (2015) foi publicada em 2015, ano no qual o estudo de Fteimi (2015) legitimou os achados dos referidos pesquisadores quanto à necessidade de um modelo holístico. Desse modo, busca-se identificar se o modelo proposto preencheu a lacuna citada.

Nesse sentido, esta pesquisa se caracteriza pelos aspectos de originalidade e relevância. É original por explorar e ajustar a GC ao paradigma holístico, sendo campos científicos caracterizados como prementes para a ciência contemporânea. E é relevante por evidenciar uma forma de conceber a GC por meio do paradigma holístico e elucidar um método para prover a análise de modelos de gerenciamento do conhecimento com essa envergadura, contribuindo para que pesquisadores desenvolvam e impulsionem essa corrente teórica.

Além desta introdução, a seção seguinte delineia os fundamentos teóricos acerca do gerenciamento do conhecimento holístico, bem como consolida as dimensões (partes) críticas para o êxito dessa gestão. Os procedimentos metodológicos adotados para o exame do modelo de Fivaz e Pretorius (2015), à luz da GC holística, são então apresentados. Na sequência, o mencionado modelo é analisado, e os resultados desse exame são expostos. Por fim, as considerações finais são relatadas, e as referências bibliográficas concluem esta pesquisa.

\section{A Gestão do Conhecimento holística e suas dimensões}

A GC holística é uma forma de abordar o gerenciamento do conhecimento para complementar a abordagem atual, visando a uma gestão mais efetiva do conhecimento organizacional. Nesse lógica, a GC holística se posiciona entre o hiato das abordagens atuais e as pretendidas para o futuro do gerenciamento do conhecimento.

A GC atual é forjada no paradigma reducionista. Esse paradigma determina que o problema seja seccionado em menores partes e que essas frações do todo sejam analisadas de maneira isolada das demais, permitindo, assim, que se compreenda o fenômeno em sua completude (Crema, 2015). Isso é aplicado nos diversos ramos da ciência desde a época de René Descartes (1596-1650), propositor desse paradigma, como apresentado de modo significativo por Capra (2014).

As organizações, locus de aplicação prática do gerenciamento do conhecimento, são sedimentadas no reducionismo desde a Administração Científica (Silveira et al., 2018; Ramalho; Locatelli; Silva, 2018), sendo fracionadas 
em organogramas, setores, áreas, cargos e funções. A GC, assim como as organizações, se segmenta em partes (dimensões), como cultura organizacional, estratégia, motivação, tecnologia da informação e outras (Natale; Neves; Carvalho, 2016; Gonzalez; Martins; Melo, 2018).

Em vista disso, o cenário atual da GC pode ser visualizado pelos modelos de gerenciamento do conhecimento, também denominados estruturas ou frameworks. Esses modelos são meios de operacionalizar a GC na prática e representam as dimensões (partes) dessa gestão e suas interfaces (Heisig, 2009). No entanto, existem diversos modelos de GC que abordam distintas dimensões (Fteimi, 2015), confirmando a redução desse fenômeno de forma disjunta, segundo os preceitos do paradigma reducionista.

A GC futura, defendida nesta pesquisa, é fundamentada nas análises de Holsapple e Joshi (1999), Rubenstein-Montano et al. (2001), Heisig (2009) e Fteimi (2015) sobre 270 modelos de GC. Essas pesquisas demarcam o paradigma holístico como uma alternativa para o desenvolvimento de frameworks orientados a uma gestão efetiva do conhecimento organizacional. O paradigma holístico diverge de seu antecessor, o reducionismo, por determinar que as partes do fenômeno sejam analisadas em conjunto. Desse modo, as conexões entre as secções (partes) são mantidas e se pode atingir o todo dessa gestão de forma completa e íntegra (Crema, 2015; Pereira, 2017).

Assim, a GC holística apresenta-se como um tipo de gestão que admite as partes existentes tanto na Administração quanto na GC, mas apregoa que estas sejam analisadas em conjunto para que as conexões adjacentes sejam mantidas de modo a atingir o hólus dessa gestão. Em outros termos, um modelo de GC holístico deve contemplar todas as dimensões (partes) do gerenciamento do conhecimento para que as conexões entre essas secções sejam mantidas e se atinja uma gestão íntegra e totalitária do conhecimento organizacional.

Uma vez que, para atingir uma gestão holística do conhecimento, é necessário abordar todas as dimensões (partes) concomitantemente, é imprescindível determinar quais são as partes que dão forma ao todo. Esse imperativo tem guarida na menção de Morin (2005, p.181), que assinala: "Pascal, que dizia: 'Só posso compreender um todo se conheço, especificamente, as partes, mas só posso compreender as partes se conhecer o todo'".

Logo, para estabelecer as partes que conformam o todo da GC holística, a corrente teórica de fatores críticos de sucesso torna-se aderente. Consequentemente, fatores críticos de sucessos resultam em áreas, denominadas partes ou dimensões de uma gestão, que, quando bem-sucedidas, garantem o êxito de uma iniciativa (Arif; Shalhoub, 2014; Zieba; Zieba, 2014; Kumar; Singer; Haleem, 2015).

No âmbito da GC, a corrente de pesquisa sobre "fatores críticos de sucesso" vem sendo explorada frequentemente (Al-Rasheed; Berri, 2017; An et al., 2017; O'Brien, 2017; Serna; Bachiller; Serna, 2017), visando identificar as áreas que são mister para o triunfo do gerenciamento do conhecimento. Portanto, se são críticas para o sucesso dessa gestão, as dimensões imperativas devem ser consideradas em um modelo de GC holístico. Conferindo crédito aos estudos que se dedicaram a essa corrente teórica, esta pesquisa consolida esses fatores (dimensões) por meio de uma revisão sistemática da literatura.

Em 2017, especificamente no dia 2 de agosto, uma busca na base Scopus foi realizada, pesquisando no título das publicações científicas os descritores "knowledge management" e "critical success factors". O total de 48 publicações foi obtido. Foram desconsiderados: estudos indisponíveis para download; que não continham os descritores no título; duplicados; e que não fundamentavam teoricamente as dimensões críticas para o sucesso da GC. Por fim, chegou-se a 15 publicações, as quais, após consolidadas por sua leitura íntegra, resultaram em 13 dimensões críticas para o êxito da GC. Por conseguinte, essas dimensões são imperativas para uma gestão holística do conhecimento, a saber:

1) Estratégia: a organização e a GC devem ter suas estratégias alinhadas para que o gerenciamento do conhecimento apoie os objetivos do negócio, e os funcionários devem ser comunicados para que compreendam e desempenhem suas atividades em prol do alinho previamente realizado (Al-Mabrouk, 2006; Gai; Xu, 2009; Altaher, 2010; Sedighi; Zand, 2012). 
2) Liderança e suporte da alta administração: a liderança deve atuar como um exemplo do comportamento esperado das pessoas, e a alta administração deve apoiar e subsidiar a proposta da GC, provendo recursos indispensáveis para a condução dessa iniciativa (Wong, 2005; Wai; Hong; Din, 2011; Arif; Shalhoub, 2014; Zieba; Zieba, 2014; Kumar; Singer; Haleem, 2015).

3) Equipe de Gestão do Conhecimento: a GC demanda uma equipe de profissionais com responsabilidades e atividades específicas, como gerenciar e coordenar o programa de gerenciamento do conhecimento, visando a sua longevidade e atingimento dos objetivos ambicionados (Wong, 2005; Al-Mabrouk, 2006; Lin; Lin, 2006; Sedighi; Zand, 2012; Arif; Shalhoub, 2014).

4) Recursos (financeiro, humano, material e tempo): a GC, como qualquer iniciativa, requer recursos financeiros e humanos, bem como necessita de insumos materiais e de tempo para a operacionalização de suas atividades (Al-Mabrouk, 2006; Gai; Xu, 2009; Abbaszadeh; Ebrahimi; Fotouhi, 2010; Sedighi; Zand, 2012; Kumar; Singer; Haleem, 2015).

5) Processos e atividades: são o centro da GC, pois anunciam o que pode ser feito com o conhecimento e determinam o ciclo de vida desse ativo, sendo identificação, armazenamento, compartilhamento e criação alguns exemplos de processos e atividades presentes em modelos de gerenciamento do conhecimento (Wong, 2005; Akhavan; Jafari; Fathian, 2006; Abbaszadeh; Ebrahimi; Fotouhi, 2010; Sedighi; Zand, 2012).

6) Gestão de recursos humanos: as pessoas são o sustentáculo da GC, visto que o conhecimento advém de suas mentes; portanto, os processos de recrutamento, desenvolvimento e retenção de funcionários municiam a empresa de profissionais com conhecimentos atinentes aos propósitos organizacionais (Wong, 2005; Valmohammadi, 2010; Anggia et al., 2013; Kumar; Singer; Haleem, 2015).

7) Treinamento e educação: os funcionários precisam ser educados quanto ao vocabulário específico do gerenciamento do conhecimento e treinados para manusear os sistemas tecnológicos adquiridos para o programa de GC (Gai; Xu, 2009; Abbaszadeh; Ebrahimi; Fotouhi, 2010; Anggia et al., 2013; Kumar; Singer; Haleem, 2015).

8) Motivação: incentivos, como recompensas financeiras e não financeiras, reconhecimento e valoração do indivíduo, são necessários para que funcionários se envolvam e pratiquem a GC de forma colaborativa (Wong, 2005; Al-Mabrouk, 2006; Lin; Lin, 2006; Valmohammadi, 2010; Sedighi; Zand, 2012; Zieba; Zieba, 2014; Arif; Shalhoub, 2014).

9) Trabalho em equipe: dois ou mais indivíduos que se influenciam e interagem em prol de um objetivo comum instituem o trabalho em equipe benéfico para o compartilhamento e a criação do conhecimento organizacional (Akhavan; Jafari; Fathian, 2006; Wai; Hong, Din; 2011; Sedighi; Zand, 2012).

10) Cultura: a GC demanda uma cultura fecunda para o estabelecimento de um alto grau de confiança mútua entre os funcionários, de modo a prover um ambiente colaborativo, que tenda a valorizar o conhecimento, sua criação, compartilhamento e aplicação no âmbito organizacional (Lin; Lin, 2006; Al-Mabrouk, 2006; Gai; Xu, 2009; Valmohammadi, 2010).

11) Tecnologia da informação: é um meio para a manipulação (captura, armazenamento e divulgação) do conhecimento explícito e um facilitador para conectar as pessoas em prol do conhecimento tácito, sendo indiscutível sua relevância para a GC (Wong, 2005; Abbaszadeh; Ebrahimi; Fotouhi, 2010; Arif; Shalhoub, 2014; Kumar; Singer; Haleem, 2015).

12) Mensuração: a medição é imprescindível para que seja possível identificar se os objetivos foram alcançados e aferir o progresso do gerenciamento do conhecimento, de modo a permitir melhorar e aperfeiçoar continuamente a iniciativa de GC (Lin; Lin, 2006; Valmohammadi, 2010; Sedighi; Zand, 2012; Arif; Shalhoub, 2014; Kumar; Singer; Haleem, 2015). 
13) Projeto-piloto: antes de pôr em prática uma iniciativa de conhecimento em âmbito organizacional, torna-se mister a implementação de projetos-piloto em menor escala para que se possam apreender as lições aprendidas e as melhores práticas, visando ao sucesso da iniciativa, posteriormente, em maior abrangência (Akhavan; Jafari; Fathian, 2006).

As treze dimensões acima descritas são assinaladas como críticas para o sucesso da GC e resultantes de pesquisas realizadas de 1997 a 2015. Portanto, devem ser abordadas em conjunto em um modelo de gerenciamento do conhecimento desenvolvido mediante os preceitos do paradigma holístico.

\section{Procedimentos Metodológicos}

Para promover o exame do modelo de Fivaze Pretorius (2015), esta pesquisa emprega, em etapas sequenciais, o método de Análise de Conteúdo, de cunho qualitativo, e a quantificação percentílica. Primeiramente, o referido modelo é analisado buscando inferir a presença das 13 dimensões da GC holística, sendo essas as unidades de registro imperativas para a aplicação da Análise de Conteúdo (Bardin, 2015). Uma vez que a abordagem qualitativa admite a subjetividade do pesquisador, serão utilizadas, frequentemente, citações diretas. Isso permite o regresso aos trechos do modelo analisado e a manutenção das inferências produzidas, imprimindo rigor na aplicação do método.

Por consequência, diante das dimensões presentes e ausentes no modelo, a quantificação percentílica simples é utilizada para aferir o nível de adesão deste à GC holística. Desse modo, torna-se possível identificar o quanto o modelo analisado adere, em pontos percentuais, ao gerenciamento do conhecimento orientado pelos preceitos do paradigma holístico.

\section{Análise e Resultados}

Segundo Fivaz e Pretorius (2015), o objetivo do estudo é propor um modelo de GC que auxilie a gestão desse ativo em ambientes de produção fabril. Para isso, os pesquisadores buscam resposta para a seguinte interrogativa: "Quais práticas de gerenciamento de conhecimento, em um ambiente de fabricação, promovem criação, compartilhamento e uso do conhecimento?" (Fivaz; Pretorius, 2015, p.2, tradução nossa)².

As práticas identificadas pelos pesquisadores foram combinadas em um modelo constituído por três blocos, sendo: (1) necessidade de conhecimento; (2) processos de GC; e (3) mensuração. No primeiro bloco, a estratégia da organização e seu desempenho se relacionam mutuamente e determinam as necessidades de conhecimento da organização. Essencialmente, os pesquisadores visualizam esses elementos pela relação: "A estratégia afeta as necessidades de conhecimento de uma planta de fabricação [...]. O desempenho de uma fábrica pode criar uma lacuna de conhecimento" (Fivaz; Pretorius, 2015, p.7, tradução nossa) ${ }^{\mathbf{3}}$.

De acordo com Fivaz e Pretorius (2015), a estratégia e o desempenho são afetados pela cultura e liderança da organização. A cultura adere-se à dimensão "Cultura" da GC holística, pois visa estabelecer um elevado grau de confiança entre os funcionários para a constituição de um ambiente colaborativo, que tende a valorizar o conhecimento, sua criação, compartilhamento e aplicação no âmbito organizacional (Lin; Lin, 2006; Al-Mabrouk, 2006; Gai; Xu, 2009; Valmohammadi, 2010). A dimensão Liderança do modelo não permite inferir sua aderência à dimensão "Liderança e suporte da alta administração" da GC holística, uma vez que não há descrição dessa dimensão no estudo dos pesquisadores.

Em continuidade, primeiramente deve-se"[...] determinar qual é a lacuna" (Fivaz; Pretorius, 2015, p.4, tradução nossa) ${ }^{4}$ de conhecimento. Em seguida, busca-se "[...] determinar [...] se há conhecimento disponível para preencher

\footnotetext{
2 No original: "Which knowledge management practices, in a manufacturing environment, promote the creation, sharing and use of knowledge?".

3 No original: "The strategy affects the knowledge need of a manufacturing plant".

4 No original: "[...] determine what the gap".
} 
ou superar a lacuna" (Fivaz; Pretorius, 2015, p.4, tradução nossa) forma a compor a fonte - local e tipo -, para sua obtenção. Assim, a necessidade de conhecimento consiste na lacuna de conhecimento, a qual resulta em determinar os conhecimentos necessários e os existentes de modo a identificar os gaps de conhecimento e ações para preencher essa lacuna.

Isso é aderente à dimensão "Estratégia" da GC holística, sob a perspectiva de alinhamento. As organizações possuem metas e objetivos particulares intrinsecamente relacionados ao seu negócio e estabelecem suas estratégias de forma a atingir o almejado. Dessa maneira, a estratégia de negócio deve estar alinhada à estratégia da GC, pois o programa de GC deve apoiar os objetivos do negócio (Al-Mabrouk, 2006; Gai; Xu, 2009; Altaher, 2010; Sedighi; Zand, 2012).

Uma vez identificada a lacuna e as fontes de conhecimento, os processos de GC (bloco 2) são postos em ação, iniciando pela determinação de "[...] como o conhecimento será obtido ou criado" (Fivaz; Pretorius, 2015, p.4, tradução nossa) ${ }^{6}$. Conforme Fivaz e Pretorius (2015), as fontes de conhecimento, as quais apontam para o tipo e o local do conhecimento, determinam "como" promover a obtenção ou criação desse ativo. Esse processo assume que o conhecimento obtido ou adquirido seja interpretado (alterado, adaptado ou internalizado) de forma a adequar-se à empresa.

Após interpretado, os pesquisadores assinalam que esse conhecimento "[...] pode ser compartilhado em um ambiente de grupo e depois usado para atender à necessidade identificada" (Fivaz; Pretorius, 2015, p.4, tradução nossa) ${ }^{7}$. Por conseguinte, a mensuração (bloco 3) visa avaliar se a lacuna de conhecimento foi abordada de forma satisfatória, pois a "[...] eficácia do conhecimento utilizado precisa refletir no desempenho da planta de fabricação" (Fivaz; Pretorius, 2015, p.4, tradução nossa) ${ }^{\mathbf{8}}$.

A mensuração (bloco 3) e o impacto dos processos de GC (bloco 2) no desempenho organizacional são aderentes à dimensão "Mensuração" da GC holística, uma vez que medir é necessário para que seja possível identificar se os objetivos foram alcançados e avaliar o seu progresso para a promoção de melhoria contínua da iniciativa de GC (Lin; Lin, 2006; Valmohammadi, 2010; Sedighi; Zand, 2012; Arif; Shalhoub, 2014; Kumar; Singer; Haleem, 2015).

Após a mensuração, o armazenamento do conhecimento deve ser realizado, por meio de sistemas de informação, para que esse ativo possa ser acessado e utilizado no futuro. Conforme os pesquisadores, "O conhecimento deve ser armazenado; mesmo se o conhecimento não funcionou em um caso específico, ele poderia adicionar algum valor no futuro para outra lacuna" (Fivaz; Pretorius, 2015, p.4, tradução nossa).

O armazenamento por sistemas de informação adere-se à dimensão "Tecnologia da informação" da GC holística, pois a tecnologia da informação é um facilitador eficaz de manipulação (captura, armazenamento e divulgação) do conhecimento explícito, e sua relevância para a GC é indiscutível (Wong, 2005; Abbaszadeh; Ebrahimi; Fotouhi, 2010; Arif; Shalhoub, 2014; Kumar; Singer; Haleem, 2015).

Os pesquisadores ponderam sobre a relevância das pessoas na organização e determinam que o "[...] objetivo principal da função de recursos humanos é cuidar das pessoas e garantir [...] apoio do pessoal em geral" (Fivaz; Pretorius, 2015, p.6, tradução nossa) ${ }^{\mathbf{1 0}}$.

Isso é aderente à dimensão "Gestão de recursos humanos" da GC holística, em razão de essa gestão ser imperativa para tratativas relacionadas ao conhecimento, uma vez que o conhecimento advém da mente das pessoas; portanto, estas são a pedra angular da GC (Wong, 2005; Valmohammadi, 2010; Anggia et al., 2013; Kumar; Singer; Haleem, 2015).

Por fim, Fivaz e Pretorius (2015), ressaltam que os processos de GC (bloco 2) de criação, compartilhamento e uso ocorrem simultaneamente na organização. Esses processos são aderentes à dimensão "Processos e atividades"

\footnotetext{
${ }^{5}$ No original: "[...] and if there is knowledge available to fill or overcome the gap".

"No original: "[...] how the knowledge is obtained or created".

7 No original: "[...] can be shared in a group environment and then used in order to fulfill the identified need".

${ }^{8}$ No original: "[...] effectiveness of the knowledge used need to reflect in the performance of the manufacturing plant".

9 No original: "The knowledge should be stored even if the knowledge did not work in a specific case it might add some value in future for another gap".

${ }^{10}$ No original: "[...] the human resource function's main purpose is to look after the people and to make sure [...] general personnel support".
} 
da GC holística, já que são o centro da GC - e do modelo dos autores -, anunciando o que pode ser feito com o conhecimento e determinando o ciclo de vida desse ativo (Wong, 2005; Akhavan; Jafari; Fathian, 2006; Abbaszadeh; Ebrahimi; Fotouhi, 2010; Sedighi; Zand, 2012).

O modelo de Fivaz e Pretorius (2015), é amplo por contemplar a GC desde a estratégia até a mensuração. Entretanto, negligencia diversas dimensões substanciais para a GC. Não considerar a "Liderança e o suporte da alta administração" e uma "Equipe de GC" pode culminar na descontinuidade da iniciativa no locus de sua aplicação. Falta de "Treinamento e educação" para que os funcionários compreendam o vocabulário da GC e manuseiem o sistema tecnológico de armazenamento do conhecimento e desatenção quanto à "Motivação" tendem ao não engajamento dos profissionais na prática diária da GC.

Por síntese, o modelo é aderente às seguintes dimensões da GC holística: (1) Estratégia: sim, pela perspectiva de alinhamento da GC ao negócio; (2) Liderança e suporte da alta administração: não especificado. A dimensão "Liderança" não é descrita no modelo, não permitindo inferir sua aderência a esta dimensão da GC holística; (3) Equipe de gestão do conhecimento: não determinado; (4) Recursos (financeiro, humano, material e tempo): não determinado; (5) Processos e atividades: sim, com criação, compartilhamento, uso e armazenamento do conhecimento; (6) Gestão de recursos humanos: sim, ponderada como imprescindível por "[...] cuidar das pessoas e garantir [...] suporte geral de pessoal" (Fivaz; Pretorius, 2015, p.6, tradução nossa) 11; ; (7) Treinamento e educação: não determinado; (8) Motivação: não determinado; (9) Trabalho em equipe: não determinado; (10) Cultura: sim, elemento que influencia a estratégia e o desempenho organizacional; (11) Tecnologia da informação: sim; (12) Mensuração: sim; e (13) Projeto-piloto: não determinado.

Mediante a análise promovida sobre o modelo de Fivaz e Pretorius (2015), as dimensões acima ponderadas são dispostas na Figura 1, que exprime a adesão do referido modelo às dimensões da GC holística concernentes a esta pesquisa. A presença de uma dimensão a posiciona na escala 1 (extremidade externa) da figura, e sua ausência a assinala na escala 0 (centro).

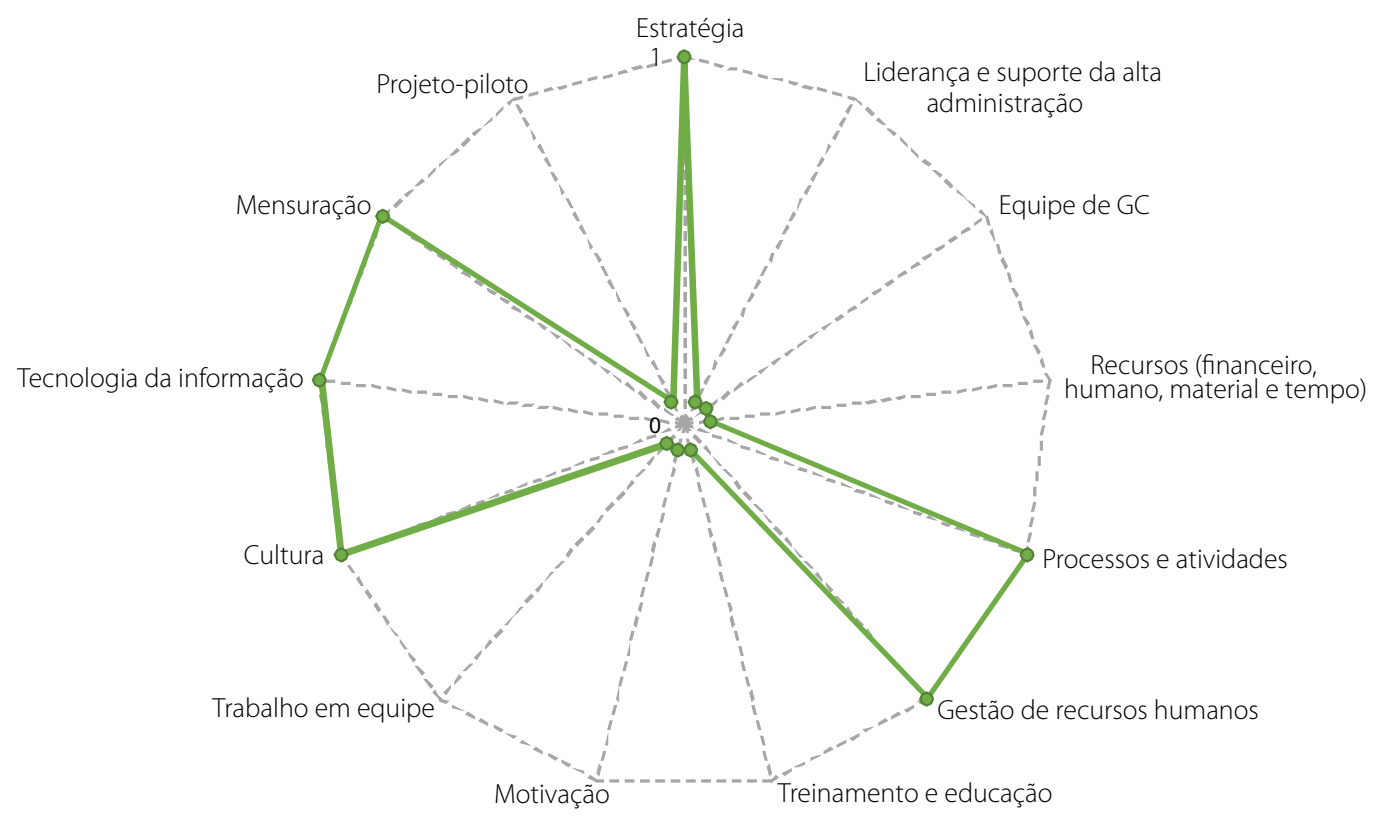

Figura 1. Adesão do modelo de Fivaz e Pretorius (2015), às dimensões da gestão do conhecimento holística.

Fonte: Elaborado pelo autor (2018).

${ }^{11}$ No original: "[...] look after the people and to make sure [...] general personnel support". 
Assim, o modelo de Fivaz e Pretorius (2015), contempla as dimensões "Estratégia, processos e atividades", "Gestão de recursos humanos", "Cultura", "Tecnologia da informação" e "Mensuração", o remetendo ao percentual de 46,2\% (6 do total de 13 dimensões) de adesão à GC holística.

\section{Conclusão}

O delineamento exposto nesta pesquisa quanto à GC holística e às dimensões que a conformam tende a estimular pesquisadores para o desenvolvimento de novos modelos, considerando todos os fatores críticos para o sucesso do gerenciamento do conhecimento. Ressalta-se que a negligência de alguma dimensão conduz à constituição de modelos ainda atinentes à realidade da atual GC, sendo necessário avançar para uma abordagem holística, anunciada como premente para o futuro do gerenciamento do conhecimento.

Conclui-se que o modelo analisado desconsidera aspectos impreteríveis para o gerenciamento do conhecimento holístico, como liderança, suporte da alta administração e equipe de GC, o que pode causar a descontinuidade da iniciativa no âmbito organizacional. Falta de treinamento e educação para que os funcionários compreendam o vocábulo da GC e façam uso da tecnologia de armazenamento do conhecimento, bem como desconsideração de aspectos motivacionais tendem à não adesão dos profissionais à GC.

Destarte, pode-se inferir que um modelo de GC fundamentado nos preceitos do paradigma holístico ainda permanece como uma lacuna científica, pois a estrutura analisada adere $46,2 \%$ a essa perspectiva. Esse resultado não é conclusivo, uma vez que requer a análise de outros modelos seguindo os delineamentos metodológicos desenvolvidos nesta pesquisa, uma oportunidade para futuras investigações.

\section{Referências}

Abbaszadeh, M. A.; Ebrahimi, M.; Fotouhi, H. Developing a causal model of critical success factors for knowledge management implementation. In: International Conference on Education and Management Technology, 2010, Cairo, Egito. Proceedings [...]. Cairo: IEEE Xplore, 2010. p. 701-705. Doi: http://dx.doi.org/10.1109/ICEMT.2010.5657563

Akhavan, P.; Jafari, M.; Fathian, M. Critical success factors of knowledge management systems: a multi-case analysis. European Business Review, v. 18, n. 2, p. 97-113, 2006. Doi: http://dx.doi.org/10.1108/09555340610651820

Al-Mabrouk, K. Critical success factors affecting knowledge management adoption: a review of the literature. In: Innovations in Information Technology, 2006, Dubai. Proceedings [...]. Dubai: IEEE Xplore, 2006. p. 1-6. Doi: http://dx.doi.org/10. 1109/INNOVATIONS.2006.301888

Al-Rasheed, A.; Berri, J. Effective reuse and sharing of best teaching practices. Computer Applications in Engineering Education, v. 25, n. 2, p. 163-178, 2017.

Altaher, A. M. Critical success factors of implementation knowledge management process. In: International Conference on Information Society, 2010, London. Proceedings [...]. London: IEEE Xplore, 2010, p. 340-348.

An, X. et al. A knowledge management framework for effective integration of national archives resources in China. Journal of Documentation, v. 73, n. 1, p. 18-34, 2017.
Anggia, P. et al. Identifying critical success factors for knowledge management implementation in organization: a survey paper. In: International Conference on Advanced Computer Science and Information Systems, 2013, Bali. Proceedings [...]. Bali: IEEE Xplore, 2013. p. 83-88. Doi: http:// dx.doi.org/10.1109/ICACSIS.2013.6761556

Arif, M. J.; Shalhoub, M. H. B. Critical success factors with its effective role in knowledge management initiatives in public and private organizations in Saudi Arabia: experts perspectives. Life Science Journal, v. 11, n. 6, p. 636-645, 2014.

Bardin, L. Análise de conteúdo. Lisboa: Edições 70, 2015.

Capra, F. A visão sistêmica da vida: uma concepção unificada e suas implicações filosóficas, políticas, sociais e econômicas. São Paulo: Cultrix, 2014.

Crema, R. Introdução à visão holística: breve relato de viagem do velho ao novo paradigma. 6. ed. São Paulo: Summus, 2015.

Fivaz, A.; Pretorius, M. W. A knowledge management framework for manufacturing firms in South Africa. In: Engineering, Technology and Innovation/International Technology Management Conference (ICE/ITMC), 2015, Belfast. Proceedings [...]. Belfast: IEEE Xplore, 2015. p. 1-9. Doi: http://dx. doi.org/10.1109/ICE.2015. 7438640

Fteimi, N. Analyzing the literature on knowledge management frameworks: towards a normative knowledge management 
classification schema. In: European Conference on Information Systems, 2015, Münster. Proceedings [...]. Münster: IEEE Xplore, 2015. p. 1-16

Gai, S.; Xu, C. Research of critical success factors for implementing knowledge management in China. In: International Conference on Information Management, Innovation Management and Industrial Engineering, 2009, Xi'na. Proceedings [...]. Xi'na: IEEE Xplore, 2009, p. 561-564. Doi: http://dx.doi.org/10.1109/ICIII.2009.594

Gonzalez, R. V. D.; Martins, M. F.; Melo, T. M. Gestão do conhecimento: tipologia a partir dos fatores contextuais da organização. Transinformação, v. 30, n. 2, p. 249-266, 2018.

Heisig, P. Harmonisation of knowledge management: comparing $160 \mathrm{KM}$ frameworks around the globe. Journal of Knowledge Management, v. 13, n. 4, p. 4-31, 2009. Doi: http:// dx.doi.org/10.1108/13673270910971798

Holsapple, C. W.; Joshi, K. D. Description and analysis of existing knowledge management frameworks. In: Annual Hawaii International Conference on Systems Sciences, 1999, Maui. Proceedings [...]. Maui: IEEE Xplore, 1999. p. 1-15. Doi: http://dx.doi.org/10.1109/HICSS.1999.772796

Kuhn, T.S. A estrutura das revoluções científicas. São Paulo: Perspectiva, 2018.

Kumar, S.; Singh, V.; Haleem, A. Critical success factors of knowledge management: modelling and comparison using various techniques. International Journal of Industrial and Systems Engineering, v. 21, n. 2, p. 180-206, 2015. Doi: http:// dx.doi.org/10.1504/IJISE.2015.071508

Lin, Y-C.; Lin, L-K. Critical success factors for knowledge management studies in construction. In: International Symposium on Robotics and Automation in Construction, 2006, Tóquio. Proceedings [...]. Tóquio: IEEE Xplore, 2006. p. 768-772.

Morin, E. Ciência com consciência. Rio de Janeiro: Bertrand Brasil, 2005.

Natale, C. H. C.; Neves, J. T. R.; Carvalho, R. B. Maturidade em gestão do conhecimento: análise das percepções dos gestores de uma grande empresa de construção civil. Informação \& Informação, v. 21, n. 1, p. 375-406, 2016.

O'Brien, J.Integrating the cultural perspective into two knowledge management frameworks. Knowledge Management, v. 17, n. 1, p. 21-35, 2017.
Pereira, M. F. A gestão organizacional em busca do comportamento holístico. In: Angeloni, M. T. Organizações do conhecimento: infra-estrutura, pessoas e tecnologia. São Paulo: Saraiva, 2017. p. 2-28.

Ramalho, W., Locatelli, R. L., Silva, S. D. C. D. Análise organizacional sob a ótica da teoria da complexidade: proposição e aplicação de um modelo. Revista Gestão \& Tecnologia, v. 18, n. 2, p. 200-223, 2018.

Rubenstein-Montano, B. et al. A systems thinking framework for knowledge management. Decision Support Systems, v. 31, n. 1, p. 5-16, 2001. Doi: http://dx.doi.org/10.1016/S0167-92 36(00)00116-0

Sedighi, M.; Zand, F. Knowledge management: review of the critical success factors and development of a conceptual classification model. In: International Conference on ICT and Knowledge Engineering, 2012, Bangkok. Proceedings [...]. Bangkok: IEEE Xplore, 2012. p. 1-9. Doi: http://dx.doi. org/10.1109/ICTKE.2012.6408553

Serna, M. E.; Bachiller, S. O.; Serna, A. A. Knowledge meaning and management in requirements engineering. International Journal of Information Management, v. 37, n. 3, p. 55-161, 2017.

Silveira, M. A. S. G. et al. Gestão de processo de compartilhamento do conhecimento tecnológico em uma empresa do setor elétrico (CEMIG). Informação \& Informação, v. 23, n. 2, p. 538-565, 2018.

Valmohammadi, C. Investigation and assessment of critical success factors of knowledge management implementation in Iranian small-to-medium sized enterprises. Journal of Applied Sciences, v. 10, n. 19, p. 2290-2296, 2010.

Wai, Y. M.; Hong, A. N. H.; Din, S. B. Critical success factors and perceived benefits of knowledge management implementation: towards a conceptual framework. Australian Journal of Basic and Applied Sciences, v. 5, n. 10, p. 754-760, 2011

Wong, K. Y. Critical success factors for implementing knowledge management in small and medium enterprises. Industrial Management \& Data Systems, v. 105, n. 3, p. 261-279, 2005. Doi: http://dx.doi.org/10.1108/02635570510590101

Zieba, M.; Zieba, K. Knowledge management critical success factors and the innovativeness of KIBS companies. Engineering Economics, v. 25, n. 4, p. 458-465, 2014. Doi: http://dx.doi. org/10.5755/j01.ee.25.4.6494 